\title{
TWIN SIGNED ROMAN DOMATIC NUMBERS IN DIGRAPHS
}

\author{
SEYED MAHMOUD SHEIKHOLESLAMI AND LUTZ VOLKMANN
}

\begin{abstract}
Let $D$ be a finite simple digraph with vertex set $V(D)$. A twin signed Roman dominating function on the digraph $D$ is a function $f: V(D) \rightarrow\{-1,1,2\}$ satisfying the conditions that (i) $\sum_{x \in N^{-}[\nu]} f(x) \geq 1$ and $\sum_{x \in N^{+}[\nu]} f(x) \geq 1$ for each $v \in V(D)$, where $N^{-}[v]$ (resp. $N^{+}[v]$ ) consists of $v$ and all in-neighbors (resp. out-neighbors) of $v$, and (ii) every vertex $u$ for which $f(u)=-1$ has an in-neighbor $v$ and an out-neighbor $w$ for which $f(\nu)=f(w)=2$. A set $\left\{f_{1}, f_{2}, \ldots, f_{d}\right\}$ of distinct twin signed Roman dominating functions on $D$ with the property that $\sum_{i=1}^{d} f_{i}(v) \leq 1$ for each $v \in V(D)$, is called a twin signed Roman dominating family (of functions) on $D$. The maximum number of functions in a twin signed Roman dominating family on $D$ is the twin signed Roman domatic number of $D$, denoted by $d_{s R}^{*}(D)$. In this paper, we initiate the study of the twin signed Roman domatic number in digraphs and we present some sharp bounds on $d_{s R}^{*}(D)$. In addition, we determine the twin signed Roman domatic number of some classes of digraphs.
\end{abstract}

\section{Introduction}

Let $D$ be a finite simple directed graph with vertex set $V(D)$ and arc set $A(D)$ (briefly $V$ and $A$ ). The integers $n=n(D)=|V(D)|$ and $m=m(D)=|A(D)|$ are the order and the size of the digraph $D$. A digraph without directed cycles of length 2 is an oriented graph. If $u v$ is an $\operatorname{arc}$ of $D$, then we also write $u \rightarrow v$, and we say that $v$ is an out-neighbor of $u$ and $u$ is an in-neighbor of $v$. For every vertex $v$, we denote the set of in-neighbors and out-neighbors of $v$ by $N^{-}(\nu)=N_{D}^{-}(\nu)$ and $N^{+}(\nu)=N_{D}^{+}(\nu)$, respectively. Let $N_{D}^{-}[\nu]=N^{-}[\nu]=N^{-}(\nu) \cup\{\nu\}$ and $N_{D}^{+}[v]=N^{+}[v]=N^{+}(\nu) \cup\{v\}$. We write $d^{+}(\nu)=d_{D}^{+}(\nu)$ for the outdegree of a vertex $v$ and $d^{-}(\nu)=d_{D}^{-}(\nu)$ for its indegree. The minimum and maximum indegree and minimum and maximum outdegree of $D$ are denoted by $\delta^{-}(D)=\delta^{-}, \Delta^{-}(D)=\Delta^{-}, \delta^{+}(D)=\delta^{+}$and $\Delta^{+}(D)=\Delta^{+}$, respectively. A digraph $D$ is $r$-out-regular if $\delta^{+}(D)=\Delta^{+}(D)=r$. In addition, let $\delta=\delta(D)=\min \left\{\delta^{+}(D), \delta^{-}(D)\right\}$ and $\Delta=\Delta(D)=\max \left\{\Delta^{+}(D), \Delta^{-}(D)\right\}$ be the minimum and maximum degree of $D$, respectively. A digraph $D$ is called regular or $r$-regular if $\delta(D)=\Delta(D)=r$. If $X \subseteq V(D)$, then $D[X]$ is the subdigraph induced by $X$. If $X \subseteq V(D)$ and $v \in V(D)$, then

Received September 5, 2016, accepted December 1, 2016. 2010 Mathematics Subject Classification. 05C69.

Key words and phrases. Twin signed Roman dominating function, twin signed Roman domination number, twin signed Roman domatic number, directed graph.

Corresponding author: Seyed Mahmoud Sheikholeslami. 
$A(X, v)$ is the set of arcs from $X$ to $v$. We denote by $A(X, Y)$ the set of arcs from a subset $X$ to a subset $Y$. We denote by $D^{-1}$ the digraph obtained from $D$ by reversing the $\operatorname{arcs}$ of $D$. For a real-valued function $f: V \longrightarrow \mathbb{R}$ the weight of $f$ is $\omega(f)=\sum_{v \in V} f(v)$, and for $S \subseteq V$, we define $f(S)=\sum_{\nu \in S} f(\nu)$, so $\omega(f)=f(V)$. Consult [7] for the notation and terminology which are not defined here.

A signed Roman dominating function (abbreviated SRDF) on $D$ is defined as a function $f: V \longrightarrow\{-1,1,2\}$ such that (i) $f\left(N^{-}[v]\right)=\sum_{x \in N^{-}[v]} f(x) \geq 1$ for each vertex $v \in V$ and (ii) every vertex $u$ for which $f(u)=-1$ has an in-neighbor $v$ for which $f(v)=2$. The signed Roman domination number $\gamma_{s R}(D)$ of $D$ is the minimum weight of an SRDF on $D$. A $\gamma_{s R}(D)$-function is a signed Roman dominating function on $D$ of weight $\gamma_{s R}(D)$. The signed Roman domination number of a digraph was introduced by Sheikholeslami and Volkmann in [5] and has been studied in $[5,6]$.

In [6], a set $\left\{f_{1}, f_{2}, \ldots, f_{d}\right\}$ of distinct signed Roman dominating functions on $D$ with the property that $\sum_{i=1}^{d} f_{i}(\nu) \leq 1$ for each $v \in V(D)$, is called a signed Roman dominating family (of functions) on $D$. The maximum number of functions in a signed Roman dominating family (SRD family) on $D$ is the signed Roman domatic number of $D$, denoted by $d_{S R}(D)$.

In [2], a signed Roman dominating function of $D$ is called a twin signed Roman dominating function (briefly TSRDF) if it also is a signed Roman dominating function of $D^{-1}$, i.e., $f\left(N^{+}[v]\right) \geq 1$ for every $v \in V$ and every vertex $u$ for which $f(u)=-1$ has an out-neighbor $v$ for which $f(v)=2$. The twin signed Roman domination number for a digraph $D$ is $\gamma_{s R}^{*}(D)=$ $\min \{\omega(f) \mid f$ is an TSRDF of $D\}$. A $\gamma_{s R}^{*}(D)$-function is a twin signed Roman dominating function on $D$ of weight $\gamma_{s R}^{*}(D)$. Since every TSRDF of $D$ is an SRDF on both $D$ and $D^{-1}$ and since the constant function 1 is an TSRDF of $D$, we have

$$
\max \left\{\gamma_{s R}(D), \gamma_{s R}\left(D^{-1}\right)\right\} \leq \gamma_{s R}^{*}(D) \leq n
$$

A set $\left\{f_{1}, f_{2}, \ldots, f_{d}\right\}$ of distinct twin signed Roman dominating functions on $D$ with the property that $\sum_{i=1}^{d} f_{i}(v) \leq 1$ for each $v \in V(D)$, is called a twin signed Roman dominating family (of functions) on $D$. The maximum number of functions in a twin signed Roman dominating family (TSRD family) on $D$ is the twin signed Roman domatic number of $D$, denoted by $d_{s R}^{*}(D)$. The twin signed Roman domatic number is well-defined and

$$
d_{s R}^{*}(D) \geq 1
$$

for all digraphs $D$, since the set consisting of the TSRDF with constant value 1 forms an TSRD family on $D$. Since every TSRD family of $D$ is an SRD family on both $D$ and $D^{-1}$, we have

$$
d_{s R}^{*}(D) \leq \min \left\{d_{s R}(D), d_{s R}\left(D^{-1}\right)\right\}
$$


In this paper, we initiate the study of the twin signed Roman domatic number in digraphs and we present some sharp bounds on $d_{s R}^{*}(D)$. In addition, we determine the twin signed Roman domatic number of some classes of digraphs.

A signed Roman dominating function (SRDF) on a graph $G=(V(G), E(G))$ is defined in [1] as a function $f: V(G) \longrightarrow\{-1,1,2\}$ such that $f(N[v])=\sum_{x \in N[v]} f(x) \geq 1$ for each vertex $v \in V$, where $N[v]$ is the closed neighborhood of $v$, and every vertex $u$ for which $f(u)=-1$ is adjacent to a vertex $v$ for which $f(v)=2$. The weight of an SRDF $f$ on $G$ is $\omega(f)=\sum_{v \in V(G)} f(\nu)$. The signed Roman domination number $\gamma_{s R}(G)$ of $G$ is the minimum weight of an SRDF on $G$. A set $\left\{f_{1}, f_{2}, \ldots, f_{d}\right\}$ of distinct SRDF on $G$ with the property that $\sum_{i=1}^{d} f_{i}(v) \leq 1$ for each $v \in V(G)$, is called in [4] a signed Roman dominating family (of functions) on $G$. The maximum number of functions in a signed Roman dominating family on $G$ is the signed Roman domatic number of $G$, denoted by $d_{s R}(G)$.

An orientation of a graph $G$ is an assignment of orientations to its edges. The associated digraph $D(G)$ of a graph $G$ is obtained by replacing each edge of $G$ by a pair of two mutually opposite oriented edges. The definitions imply the next observation immediately.

Observation 1. If $G$ is a graph and $D(G)$ its associated digraph, then $\gamma_{s R}(G)=\gamma_{s R}^{*}(D(G))$ and $d_{s R}(G)=d_{s R}^{*}(D(G))$.

We make use of the following results in this paper.

Observation 2. ([1]) If $K_{n}$ is the complete graph of order $n \geq 1$, then $\gamma_{s R}\left(K_{n}\right)=1$, unless $n=3$ in which case $\gamma_{s R}\left(K_{n}\right)=2$.

Observation 3. ([4]) If $K_{n}$ is the complete graph of order $n \geq 1$, then $d_{s R}\left(K_{n}\right)=n$, unless $n=3$ in which case $d_{s R}\left(K_{n}\right)=1$.

Observations 1, 2 and 3 lead to the next results immediately.

Observation 4. If $K_{n}^{*}$ is the complete digraph of order $n \geq 1$, then $\gamma_{s R}^{*}\left(K_{n}^{*}\right)=1$, unless $n=3$ in which case $\gamma_{s R}^{*}\left(K_{n}^{*}\right)=2$.

Observation 5. If $K_{n}^{*}$ is the complete digraph of order $n \geq 1$, then $d_{s R}^{*}\left(K_{n}^{*}\right)=n$, unless $n=3$ in which case $d_{s R}^{*}\left(K_{n}^{*}\right)=1$.

If $n \geq 4$ and $\left\{f_{1}, f_{2}, \ldots, f_{n}\right\}$ is a signed Roman dominating family of functions on $K_{n}^{*}$, then we conclude from

$$
n=n \cdot 1 \leq \sum_{i=1}^{n} \omega\left(f_{i}\right)=\sum_{i=1}^{n} \sum_{v \in V\left(K_{n}^{*}\right)} f_{i}(v)=\sum_{\nu \in V\left(K_{n}^{*}\right)} \sum_{i=1}^{n} f_{i}(v) \leq \sum_{\nu \in V\left(K_{n}^{*}\right)} 1=n
$$

that $\omega\left(f_{i}\right)=1$ and so $f_{i}$ is a $\gamma_{s R}\left(K_{n}^{*}\right)$-function for each $i$. It follows that each $f_{i}$ assigns 2 to some vertex of $K_{n}^{*}$. 
Observation 6. ([3]) If $K_{p, p}$ is the complete bipartite graph of order $2 p$, then $\gamma_{s R}\left(K_{p, p}\right)=4$ when $p \geq 3$.

Using Observations 1 and 6, we obtain the next result.

Observation 7. If $K_{p, p}^{*}$ is the complete bipartite digraph of order $2 p$, then $\gamma_{s R}^{*}\left(K_{p, p}^{*}\right)=4$ when $p \geq 3$.

Observation 8. ([2]) If $C_{n}$ is an oriented cycle of order $n \geq 2$, then $\gamma_{s R}^{*}\left(C_{n}\right)=n / 2$ when $n$ is even and $\gamma_{s R}^{*}\left(C_{n}\right)=(n+3) / 2$ when $n$ is odd.

Observation 9. ([6]) If $D$ is a digraph, then $d_{s R}(D) \leq \delta^{-}(D)+1$.

Observation 10. ([6]) Let $D$ be an $r$-out-regular digraph of order $n$ such that $r \geq 1$. If $n \not \equiv$ $0(\bmod (r+1))$, then $d_{s R}(D) \leq r$.

Inequality (3) and Observation 10 imply the next corollary.

Corollary 11. Let $D$ be an $r$-out-regular digraph of order $n$ such that $r \geq 1$. If $n \not \equiv 0(\bmod (r+$ 1)), then $d_{s R}^{*}(D) \leq r$.

\section{Properties of the twin signed Roman domatic number}

In this section we present basic properties of $d_{s R}^{*}(D)$ and sharp bounds on the twin signed Roman domatic number of digraphs. Using Observation 9 and (3), we obtain our first bound on $d_{s R}^{*}(D)$.

Proposition 12. If $D$ is a digraph, then $d_{s R}^{*}(D) \leq \delta(D)+1$.

Observation 5 shows that Proposition 12 is sharp. Inequality (2) and Proposition 12 imply the next corollary immediately.

Corollary 13. If $D$ is a digraph with $\delta(D)=0$, then $d_{s R}^{*}(D)=1$.

As we observed in (3), $d_{s R}^{*}(D) \leq d_{s R}(D)$. Now, we show that the difference $d_{s R}(D)-d_{s R}^{*}(D)$ can be arbitrarily large.

Theorem 14. For every positive integer $k \geq 3$, there exists a digraph D such that

$$
d_{s R}(D)-d_{s R}^{*}(D) \geq k .
$$


Proof. Let $k \geq 3$ be an integer, and let $D$ be the digraph obtained from two copies of $K_{k+1}^{*}$, say $G_{1}, G_{2}$, by adding a new vertex $x$ and adding arcs going from every vertex in $V\left(G_{1}\right) \cup V\left(G_{2}\right)$ into $x$. Since $d^{+}(x)=0$, we deduce from Corollary 13 that $d_{s R}^{*}(D)=1$.

Let $\left\{f_{1}, f_{2}, \ldots, f_{k+1}\right\}$ be a signed Roman dominating family on the digraph $G_{1}$, and let $\left\{g_{1}, g_{2}, \ldots, g_{k+1}\right\}$ be a signed Roman dominating family on $G_{2}$. As we note after Observation 5, each $f_{i}$ assigns 2 to some vertex of $G_{1}$ and each $g_{j}$ assigns 2 to some vertex of $G_{2}$. For $1 \leq i \leq k+1$, define $h_{i}: V(D) \rightarrow\{-1,1,2\}$ by $h_{i}(x)=-1, h_{i}(u)=f_{i}(u)$ if $u \in V\left(G_{1}\right)$ and $h_{i}(u)=g_{i}(u)$ if $u \in V\left(G_{2}\right)$. Clearly, $\left\{h_{1}, h_{2}, \ldots, h_{k+1}\right\}$ is a signed Roman dominating family of $D$ and hence $d_{s R}(D) \geq k+1$. Thus $d_{s R}(D)-d_{s R}^{*}(D) \geq k$, and the proof is complete.

Theorem 15. If $D$ is a digraph of order $n$, then

$$
\gamma_{s R}^{*}(D) \cdot d_{s R}^{*}(D) \leq n
$$

Moreover, if $\gamma_{s R}^{*}(D) \cdot d_{s R}^{*}(D)=n$, then for each TSRD family $\left\{f_{1}, f_{2}, \ldots, f_{d}\right\}$ on $D$ with $d=d_{s R}^{*}(D)$, each function $f_{i}$ is a $\gamma_{s R}^{*}(D)$-function and $\sum_{i=1}^{d} f_{i}(v)=1$ for each $v \in V(D)$.

Proof. Let $\left\{f_{1}, f_{2}, \ldots, f_{d}\right\}$ be an TSRD family on $D$ with $d=d_{s R}^{*}(D)$ and let $v \in V(D)$. Then

$$
d \cdot \gamma_{s R}^{*}(D)=\sum_{i=1}^{d} \gamma_{s R}^{*}(D) \leq \sum_{i=1}^{d} \sum_{\nu \in V(D)} f_{i}(\nu)=\sum_{\nu \in V(D)} \sum_{i=1}^{d} f_{i}(v) \leq \sum_{\nu \in V(D)} 1=n .
$$

If $\gamma_{s R}^{*}(D) \cdot d_{s R}^{*}(D)=n$, then the two inequalities occuring in (4) become equalities. Hence for the TSRD family $\left\{f_{1}, f_{2}, \ldots, f_{d}\right\}$ on $D$ and for each $i, \sum_{\nu \in V(D)} f_{i}(v)=\gamma_{s R}^{*}(D)$. Thus each function $f_{i}$ is a $\gamma_{s R}^{*}(D)$-function, and $\sum_{i=1}^{d} f_{i}(v)=1$ for each $v \in V(D)$.

Observations 4 and 5 demonstrate that Theorem 15 is sharp. In [6], we have shown that $d_{s R}\left(K_{p, p}^{*}\right)=\frac{p}{2}$ when $p \geq 4$ is an even integer with $p \neq 6$. Analogously, one can prove that $d_{s R}^{*}\left(K_{p, p}^{*}\right)=\frac{p}{2}$ when $p \geq 4$ is an even integer with $p \neq 6$. Using this identity and Observation 7 , we have a further example which shows the sharpness of Theorem 15.

Applying Observation 8, Proposition 12 and Theorem 15, we obtain the twin signed Roman domatic number for oriented cycles.

Corollary 16. Let $C_{n}$ be an oriented cycle of length $n \geq 2$. Then $d_{s R}^{*}\left(C_{n}\right)=1$ when $n$ is odd and $d_{s R}^{*}\left(C_{n}\right)=2$ when $n$ is even.

Proof. First let $n$ be odd. Using Observation 8 and Theorem 15, we deduce that

$$
d_{s R}^{*}\left(C_{n}\right) \leq \frac{n}{\gamma_{s R}^{*}\left(C_{n}\right)}=\frac{2 n}{n+3}<2
$$

and thus $d_{s R}^{*}\left(C_{n}\right)=1$. 
Now let $n=2 p$ be even, and let $C_{n}=u_{1} v_{1} u_{2} v_{2} \ldots u_{p} v_{p} u_{1}$. Define the function $f_{i}: V\left(C_{n}\right) \longrightarrow$ $\{-1,1,2\}$ by $f_{1}\left(u_{i}\right)=-1$ and $f_{1}\left(v_{i}\right)=2$ and $f_{2}\left(u_{i}\right)=2$ and $f_{2}\left(v_{i}\right)=-1$ for $1 \leq i \leq p$. Then $f_{1}$ and $f_{2}$ are TSRDF on $C_{n}$ such that $f_{1}(x)+f_{2}(x)=1$ for each $x \in V\left(C_{n}\right)$. Therefore $d_{s R}^{*}\left(C_{n}\right) \geq 2$. It follows from Proposition 12 hat $d_{s R}^{*}\left(C_{n}\right) \leq 2$ and so $d_{s R}^{*}\left(C_{n}\right)=2$ when $n$ is even.

According to Corollary 16, the oriented cycle $C_{n}$ is another example which shows the sharpness of Theorem 15, when $n$ is even.

Theorem 17. If $D$ is a digraph of order $n$, then

$$
\gamma_{s R}^{*}(D)+d_{s R}^{*}(D) \leq n+1
$$

with equality if and only if $D=K_{n}^{*}(n \neq 3)$ or $\gamma_{s R}^{*}(D)=n$ and $d_{s R}^{*}(D)=1$.

Proof. It follows from Theorem 15 that

$$
\gamma_{s R}^{*}(D)+d_{s R}^{*}(D) \leq \frac{n}{d_{s R}^{*}(G)}+d_{s R}^{*}(D) .
$$

According to (2) and Proposition 12, we have $1 \leq d_{s R}^{*}(G) \leq n$. Using these bounds, and the fact that the function $g(x)=x+n / x$ is decreasing for $1 \leq x \leq \sqrt{n}$ and increasing for $\sqrt{n} \leq x \leq n$, we observe that the maximum of $g$ on the interval $[1, n]$ is $n+1$. Therefore (5) leads to the desired bound.

If $D=K_{n}^{*}(n \neq 3)$, then we deduce from Observations 4 and 5 that $\gamma_{s R}^{*}(D)+d_{s R}^{*}(D)=n+1$. Clearly, if $\gamma_{s R}^{*}(D)=n$ and $d_{s R}^{*}(D)=1$, then $\gamma_{s R}^{*}(D)+d_{s R}^{*}(D)=n+1$.

Conversely, assume that $\gamma_{s R}^{*}(D)+d_{s R}^{*}(D)=n+1$. Since the maximum of $g$ on $[1, n]$ is achieved only at 1 and $n$, it follows from (5) that

$$
n+1=\gamma_{s R}^{*}(D)+d_{s R}^{*}(D) \leq \frac{n}{d_{s R}^{*}(G)}+d_{s R}^{*}(D) \leq n+1,
$$

which implies that $\gamma_{s R}^{*}(D)=n$ and $d_{s R}^{*}(D)=1$ or $\gamma_{s R}^{*}(D)=1$ and $d_{s R}^{*}(D)=n$. If $d_{s R}^{*}(D)=n$ and $\gamma_{s R}^{*}(D)=1$, then Proposition 12 implies that $\delta(D)=n-1$ and hence $D$ is the complete digraph $K_{n}^{*}$. Since $\gamma_{s R}^{*}(D)=1$, we conclude from Observation 4 that $n \neq 3$ and so $D=K_{n}^{*}(n \neq 3)$.

If $H$ is the disjoint union of oriented triangles, then it follows from Observation 8 and Corollary 16 that $\gamma_{s R}^{*}(H)=n$ and $d_{s R}^{*}(H)=1$. Thus, in Theorem $17, \gamma_{s R}^{*}(D)=n$ and $d_{s R}^{*}(D)=1$ is possible.

The complement $\bar{D}$ of a digraph $D$ is the digraph with vertex set $V(D)$ such that for any two distinct vertices $u, v$ the arc $(u, v)$ belongs to $\bar{D}$ if and only if $(u, v)$ does not belong to $D$.

Theorem 18. For every digraph $D$ of order $n$,

$$
d_{s R}^{*}(D)+d_{s R}^{*}(\bar{D}) \leq n+1
$$

with equality if and only if $D=K_{n}^{*}$ or $\bar{D}=K_{n}^{*}$ and $n \neq 3$. 
Proof. Since $\delta(\bar{D})=n-1-\Delta(D)$, it follows from Proposition 12 that

$$
\begin{aligned}
d_{s R}^{*}(D)+d_{s R}^{*}(\bar{D}) & \leq(\delta(D)+1)+(\delta(\bar{D})+1) \\
& =(\delta(D)+1)+(n-1-\Delta(D)+1) \leq n+1,
\end{aligned}
$$

and this is the desired inequality. If $D$ is not regular, then $\Delta(D)-\delta(D) \geq 1$, and hence the above inequality chain implies the better bound $d_{s R}^{*}(D)+d_{s R}^{*}(\bar{D}) \leq n$.

If $D=K_{n}^{*}(n \neq 3)$, then we deduce from Observation 5 and Corollary 13 that $d_{s R}^{*}(D)+$ $d_{s R}^{*}(\bar{D})=n+1$.

Now assume that $d_{s R}^{*}(D)+d_{s R}^{*}(\bar{D})=n+1$. As seen above, this condition shows that $D$ is an $r$-regular digraph. Therefore $\bar{D}$ is $(n-r-1)$-regular. If $r=0$ or $r=n-1$, then $D=K_{n}^{*}$ or $\bar{D}=K_{n}^{*}$, and we obtain the desired result.

Next assume that $1 \leq r \leq n-2$ and $1 \leq \delta(\bar{D}) \leq n-2$. We assume, without loss of generality, that $r \leq(n-1) / 2$. If $n \not \equiv 0(\bmod (r+1))$, then it follows from Corollary 11 and Proposition 12 that

$$
n+1=d_{s R}^{*}(D)+d_{s R}^{*}(\bar{D}) \leq r+(n-1-r+1)=n,
$$

a contradiction. Next assume that $n \equiv 0(\bmod (r+1))$. Then $n=p(r+1)$ with an integer $p \geq 2$. If $n \not \equiv 0(\bmod (n-r))$, then it follows from Corollary 11 and Proposition 12 that

$$
n+1=d_{s R}^{*}(D)+d_{s R}^{*}(\bar{D}) \leq(r+1)+(n-1-r)=n,
$$

a contradiction. Therefore assume that $n \equiv 0(\bmod (n-r))$. Then $n=q(n-r)$ with an integer $q \geq 2$. Since $r \leq(n-1) / 2$, this leads to the contradiction

$$
n=q(n-r) \geq q\left(n-\frac{n-1}{2}\right)=\frac{q(n+1)}{2} \geq n+1,
$$

and the proof is complete.

For some special cases we will improve Proposition 12.

Theorem 19. Let $D$ be a digraph. If $D$ has a vertex $v$ with the property that $d^{+}(v)=2$ or $d^{-}(\nu)=2$, then $d_{s R}^{*}(D)=1$.

Proof. Assume, without loss of generality, that $d^{+}(v)=2$. Let $u_{1}$ and $u_{2}$ be the two outneighbors of $v$. Using Proposition 12, we observe that $d_{s R}^{*}(D) \leq 3$. First we show that $d_{s R}^{*}(D) \leq$ 2.

Suppose, to the contrary, that $d_{s R}^{*}(D)=3$, and let $\{f, g, h\}$ be a TSRD family on $D$. Since $f(x)+g(x)+h(x) \leq 1$ for each $x \in V(D)$, we deduce that $f(x)=-1$ or $g(x)=-1$ or $h(x)=-1$ for each $x \in V(D)$. In addition, if $f(y)=2$ for a vertex $y$, then $g(y)=h(y)=-1$. Now assume, 
without loss of generality, that $f(v)=-1$. Then $f\left(u_{1}\right)=2$ or $f\left(u_{2}\right)=2$, say $f\left(u_{1}\right)=2$. If $f\left(u_{2}\right)=$ -1 , then $f\left(N^{+}[v]\right)=0$, a contradiction. Next let $f\left(u_{2}\right) \geq 1$. Then, without loss of generality, $g\left(u_{2}\right)=-1$. Since $g\left(u_{1}\right)=-1$, we obtain the contradiction $g\left(N^{+}[v]\right) \leq 0$. Thus $d_{s R}^{*}(D) \leq 2$.

Next we show that $d_{s R}^{*}(D)=1$. Suppose, to the contrary, that $d_{s R}^{*}(D)=2$, and let $\{f, g\}$ be a TSRD family on $D$. Since $f(x)+g(x) \leq 1$ for each $x \in V(D)$, we deduce that $f(x)=-1$ or $g(x)=-1$ for each $x \in V(D)$. Assume, without loss of generality, that $f(v)=-1$. Then $f\left(u_{1}\right)=2$ or $f\left(u_{2}\right)=2$, say $f\left(u_{1}\right)=2$. If $f\left(u_{2}\right)=-1$, then $f\left(N^{+}[v]\right)=0$, a contradiction. Next let $f\left(u_{2}\right) \geq 1$. Then $g\left(u_{1}\right)=g\left(u_{2}\right)=-1$, and we arrive at the contradiction $g\left(N^{+}[v]\right) \leq 0$.

For $r=2$, Theorem 19 yields to the following improvement of Corollary 11 .

Corollary 20. If $D$ is a 2-out-regular digraph, then $d_{s R}^{*}(D)=1$.

Corollary 21. Let $D$ be a digraph. If $D$ has a vertex $v$ with the property that $d^{+}(v)+d^{-}(v)=3$, then $d_{s R}^{*}(D)=1$.

Proof. If $\delta(D)=0$, then Corollary 13 implies the desired result. Let now $\delta(D) \geq 1$. Since $d^{+}(v)+d^{-}(v)=3$, we observe that $d^{+}(\nu)=2$ or $d^{-}(v)=2$. Now we deduce from Theorem 19 that $d_{s R}^{*}(D)=1$.

A fan and a wheel is a graph obtained from a path and a cycle by adding a new vertex and edges joining it to all vertices of the path and cycle, respectively. Corollary 21 leads to the next result immediately.

Corollary 22. If $D$ is an orientation of a fan, a wheel or a cubic graph, then $d_{s R}^{*}(D)=1$.

\section{References}

[1] H. Abdollahzadeh Ahangar, M. A. Henning, Y. Zhao, C. Löwenstein and V. Samodivkin, Signed Roman domination in graphs, J. Comb. Optim., 27 (2014), 241-255.

[2] A. Bodaghli, S. M. Sheikholeslami and L. Volkmann, Twin signed Roman domination numbers in directed graphs, Tamkang J. Math., 47 (2016), 357-371.

[3] M. A. Henning and L. Volkmann, Signed Roman k-domination in graphs, Graphs Combin., 32 (2016), 175190.

[4] S. M. Sheikholeslami and L. Volkmann, The signed Roman domatic number of a graph, Ann. Math. Inform., 40 (2012), 105-112.

[5] S. M. Sheikholeslami and L. Volkmann, Signed Roman domination in digraphs, J. Comb. Optim., 30 (2015), 456-467.

[6] S. M. Sheikholeslami and L. Volkmann, The signed Roman domatic number of a digraph, Electro. J. Graph Theory Appl. 3 (2015), 85-93.

[7] D. B. West, Introduction to Graph Theory, Prentice-Hall, Inc, 2000.

Department of Mathematics, Azarbaijan Shahid Madani University, Tabriz, I.R. Iran.

E-mail: s.m.sheikholeslami@azaruniv.edu

Lehrstuhl II für Mathematik, RWTH Aachen University, 52056 Aachen, Germany.

E-mail: volkm@math2.rwth-aachen.de 